\title{
Structure-based Design of Prefusion-stabilized SARS-CoV-2 Spikes
}

\author{
J McLellan ${ }^{1}$ \\ ${ }^{1}$ Department of Molecular Biosciences, The University of Texas at Austin, Austin TX USA \\ jmclellan@austin.utexas.edu
}

The COVID-19 pandemic caused by the novel coronavirus SARS-CoV-2 has led to accelerated efforts to develop therapeutics, diagnostics, and vaccines to mitigate this public health emergency. A key target of these efforts is the spike (S) protein, a large trimeric class I fusion protein that is metastable and difficult to produce recombinantly in large quantities. Here, we designed and expressed over 100 structure-guided spike variants based upon a previously determined cryo-EM structure of the prefusion SARS-CoV-2 spike. Biochemical, biophysical and structural characterization of these variants identified numerous individual substitutions that increased protein yields and stability. The best variant, HexaPro, has six beneficial proline substitutions leading to $\sim 10$-fold higher expression than its parental construct and is able to withstand heat stress, storage at room temperature, and multiple freezethaws. A 3.2 Å-resolution cryo-EM structure of HexaPro confirmed that it retains the prefusion spike conformation. High-yield production of a stabilized prefusion spike protein will accelerate the development of vaccines and serological diagnostics for SARS-CoV-2.

Acta Cryst. (2020). A76, a209 